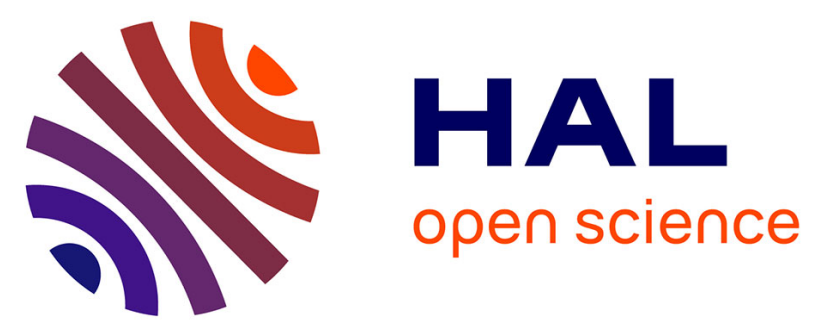

\title{
Relatório 06 - A Mortalidade por COVID-19 nos Estados Brasileiros: Uma análise comparativa entre 2019 e 2020 até o mês de maio
}

\author{
Lisandro Lovisolo, Diego H S Catalão, Americo Cunha Jr, Rodrigo Burgos, \\ Adriano Cortês, Karla Figueiredo, Malú Grave, Roberto Velho, Bruna Silveira \\ Pavlack, Diego Matos, et al.
}

\section{To cite this version:}

Lisandro Lovisolo, Diego H S Catalão, Americo Cunha Jr, Rodrigo Burgos, Adriano Cortês, et al.. Relatório 06 - A Mortalidade por COVID-19 nos Estados Brasileiros: Uma análise comparativa entre 2019 e 2020 até o mês de maio. [Technical Report] COVID-19: Observatório Fluminense. 2020. hal-02887106

\section{HAL Id: hal-02887106 https://hal.science/hal-02887106}

Submitted on 2 Jul 2020

HAL is a multi-disciplinary open access archive for the deposit and dissemination of scientific research documents, whether they are published or not. The documents may come from teaching and research institutions in France or abroad, or from public or private research centers.
L'archive ouverte pluridisciplinaire HAL, est destinée au dépôt et à la diffusion de documents scientifiques de niveau recherche, publiés ou non, émanant des établissements d'enseignement et de recherche français ou étrangers, des laboratoires publics ou privés.

\section{(ㅇ)(1) $\$$}

Distributed under a Creative Commons Attribution - NonCommercial - NoDerivatives $\mid 4.0$ 


\section{O.FID-I 9 OBSERVATÓRIO FLUMINENSE \\ www.covid19rj.org}

\section{Relatório 06 \\ A Mortalidade por COVID-19 nos Estados Brasileiros \\ Uma análise comparativa entre 2019 e 2020 até o mês de maio}

Lisandro Lovisolo*, Diego H. S. Catalão *, Americo Cunha Jr*, Rodrigo Burgos*, Adriano Cortês, Karla

Figueiredo, Malú Grave, Roberto Velho, Bruna Pavlack, Diego Matos, Eber Dantas, Gabrielle Pereira, João

Pedro Norenberg, Julio Basilio, Leonardo de la Roca, Lucas Chaves, Luiz F. S. Coelho, Marcos Issa, Michel

Tosin, Roberto Luo, Amanda Cunha Guyt, Luthiana Soares

*Contribuíram igualmente para elaboração deste relatório.

Os autores declaram nenhum conflito de interesse.

Correspondência: americo@ime.uerj.br, lisandro@uerj.br, rburgos@eng.uerj.br

Rio de Janeiro, 20 de junho de 2020

Sugestão de citação:

L. Lovisolo, et al. Relatório 06 A Mortalidade por COVID-19 nos Estados Brasileiros: Uma análise comparativa entre 2019 e 2020 até o mês de maio. COVID-19: Observatório Fluminense (20/06/2020), DOI: https://doi.org/10.12957/eduerj.covid19rj.relatorio6 


\section{COVID-19: Observatório Fluminense}

Esta é uma inciativa independente de pesquisadores, que congrega uma equipe multidisciplinar (matemática, engenharias, computação, arquitetura, jornalismo), para responder algumas demandas emergentes com o avanço da pandemia de COVID-19 em âmbito nacional. O interesse individual dos membros da equipe pela pandemia levou, naturalmente, ao intercâmbio de informações entre pesquisadores e estudantes, tais como fontes de dados, análises gráficas, notícias, relatórios e artigos científicos e, especialmente, ferramentas matemáticas empregadas na modelagem e análise do progresso de epidemias. Essa interação resultou na organização de uma força tarefa para buscar algumas soluções em termos de análise e visualização de dados, modelagem matemática da epidemia, bem como para produzir material educacional para estudantes interessados no tema e para o público em geral.

Dessa forma, os objetivos da presente iniciativa se articulam em tornos dos seguintes tópicos:

- Monitorar, em âmbito nacional e no estado do Rio de Janeiro, o progresso da pandemia de COVID-19;

- Construir gráficos e outras entidades para visualização de dados que permitam acompanhar e analisar o progresso da pandemia de modo claro e pedagógico;

- Fazer previsões confiáveis sobre o progresso de curto prazo da pandemia (número de infectados, número de óbitos, variações dos mesmos etc);

- Desenvolver material educativo de alto nível na área de modelagem matemática de epidemias;

- Desenvolver e divulgar material informativo de qualidade para o público interessado.

Mais informações sobre a iniciativa podem ser obtidas em www.covid19rj.org. Todos os gráficos e informações apresentados neste relatório, bem como o mesmo demais documentos produzidos pela equipe COVID19RJ, podem ser encontrados no repositório https://github.com/americocunhajr/COVID19RJ.

Outras informações e resultados relevantes também podem ser vistos nas redes sociais da iniciativa:

wWW. instagram.com/portalcovid19rj

WWW.facebook.com/portalcovid19rj

WW. twitter.com/portalcovid19rj 


\section{Equipe de trabalho}

\section{Professores / Pesquisadores:}

Adriano Cortês

(UFRJ)

Americo Cunha

(UERJ)

(UERJ)

(UERJ)

Lisandro Lovisolo

Malú Grave

(UFRJ)

(UFRGS)

Roberto M. Velho

Rodrigo Burgos

\section{Estudantes:}

Bruna Pavlack

Diego H.S. Catalão

Diego Matos

Eber Dantas

Gabrielle Pereira

João P. Norenberg

Julio Basilio

Leonardo de la Roca

Lucas Chaves

Luiz F. S. Coelho

Marcos Issa

Michel Tosin

Roberto Luo

\section{Design Gráfico:}

Amanda Cunha Guyt

(CCSF)

(IFMS)

(UFF)

(UERJ)

(UFRJ)

(UERJ)

(UNESP)

(UERJ)

(UERJ)

(UFU)

(UERJ)

(UERJ)

(UERJ)

(UERJ)

\section{Comunicação:}

Luthiana Soares adriano@caxias.ufrj.br

americo@ime.uerj.br

karla.figueiredo@gmail.com

lisandro@uerj.br

malugrave@nacad.ufrj.br

roberto.velho@gmail.com

rburgos@eng.uerj.br

bruna.pavlack@ifms.edu.br

diegocatalao@id.uff.br

diego.matos@uerj.br

eberdantas@ufrj.br

pereira.gabrielle@graduacao.uerj.br

p.norenberg@unesp.br

basilio.julio@posgraduacao.uerj.br

delaroca@protonmail.com

Lucasfernando@ufu.br

Ifscoelho@ieee.org

marcos.issa@uerj.br

michel.tosin@uerj.br

cai.roberto@graduacao.uerj.br

aguyt@mail.ccsf.edu

luthianassoares@gmail.com 


\section{Resumo}

Este relatório elaborado pela iniciativa COVID-19: Observatório Fluminense (COVID19RJ) reporta a mortalidade da pandemia de COVID-19 nos diferentes estados brasileiros até o mês de maio de 2020. As análises e conclusões apresentadas resultam da comparação entre o número de mortes por doenças respiratórias no presente ano (2020) com o ano passado (2019). As principais conclusões deste estudo são apresentadas no corpo desse relatório, bem como as tabelas sintéticas.

\section{Contestação de Responsabilidade}

Os resultados apresentados neste relatório resultam de simulações computacionais e análises estatísticas conduzidas com auxílio de diversos tipos de modelo matemático, que utilizam informações de várias bases de dados. A qualidade dos resultados e confiança nos valores apresentados deriva diretamente da qualidade, completude, consistência, e acurácia das fontes empregadas. Assim sendo, eventuais erros e imprecisões podem ocorrer nas análises, independentemente dos rigores técnico-científico e ético seguidos pela equipe COVID-19: Observatório Fluminense. 


\section{Metodologia}

Para avaliar o impacto da mortalidade da COVID-19 na sociedade brasileira, utilizamos os dados do Portal da Transparência de Registro Civil [1]. Devido aos atrasos existentes entre óbitos e seus registros, os dados do portal da transparência de registro civil não podem ser considerados em tempo real (e não vai aqui uma crítica, apenas uma constatação). Assim, comparamos os números de óbitos nos meses de janeiro a maio de 2020 aos de 20191

A partir desses dados acompanhamos os números de óbitos totais e devidos a causas respiratórias. EscoIhemos agrupar as diferentes causas respiratórias num único índice de forma a reduzir impactos de possíveis notificações cruzadas de óbitos devidos a diferentes causas respiratórias, seguindo a metodologia que introduzimos em [2]. De forma a considerar as sazonalidades, as agregações são realizadas por mês.

\subsection{Fontes de dados}

Os dados referentes óbitos por diferentes razões (além da COVID-19) advêm do Portal da Transparência de Registro Civil [1]:

https://transparencia.registrocivil.org.br/

\footnotetext{
${ }^{1}$ Só podemos fazer essa comparação entre os meses nesses dois anos pois os dados até 2018, disponibilizados pelo IBGE, quando comparados à série da portal transparência registro civil apresentam uma alteração de patamar em quase todos os estados e não as taxas de crescimento anual superior à esperada $(0,8-1,0)$.
} 


\section{Dados de mortalidade conforme o Registro Civil}

Esta seção apresenta uma análise comparativa da evolução do número de óbitos, em âmbito nacional e dos estados da federação, dos primeiros meses de 2020 tendo o ano de 2019 como referência, estratificando as mortes causadas por diferentes doenças respiratórias. Os dados foram obtidos do portal da transparência, site de livre acesso que disponibiliza informações sobre nascimentos, casamentos e óbitos. É importante ressaltar que tais informações apresentam certo atraso até aparecerem no site, algo em torno de 15 dias, devido aos prazos legais envolvidos no processo.

A Figura 1 apresenta um comparativo dos óbitos no Brasil entre os meses de janeiro e maio de 2020 relativamente ao ano de 2019. Os gráficos à esquerda apresentam a evolução das quantidades de óbitos, pelas principais causas respiratórias, entre os meses de janeiro e maio de 2019 (acima) e 2020 (abaixo). O gráfico à direita apresenta o total de mortes por essas causas comparativamente ao total geral de mortes (por todas as causas) no Brasil. Vemos que em abril e maio de 2020 há não só um aumento no número de óbitos por COVID-19 mas também no número de mortes atribuídas a SRAG (Síndrome Respiratória Aguda Grave), que não são observadas em 2019. Há também uma leve queda no número de mortes por pneumonia em abril de 2020 que se acentua em maio do mesmo ano e um leve aumento no número de mortes por insuficiência respiratória em abril de 2020, sendo em maio de 2020 muito similar ao observado em maio de 2019.

Nas curvas no canto superior direito da Figura 1 vemos um aumento crescente no número de óbitos por doenças respiratórias em abril de 2020, que é refletido no número de óbitos totais. Aumento esse que se torna bastante significativo em maio de 2020. Mais especificamente, o número mortes em abril de 2020 foi 9,76\% superior em relação ao de abril de 2019 , enquanto, em maio, o aumento foi de $20,63 \%$. Percebe-se claramente pelos gráficos que esses aumentos se devem a causas respiratórias, já que o número de mortes por causas respiratórias aumentou em 36,19\% em abril e em 77,92\% em maio de 2020 comparativamente aos mesmos meses do ano anterior no Brasil.

Em abril, acompanhamos notícias indicando os estados do AM, CE, MA, PA, PE, RJ e SP como muito infectados pela COVID-19. A partir dos dados acima discutidos, podemos calcular que nesses estados, O número de óbitos cresceu 15,5\% em abril de 2020 relativamente a abril de 2019 , enquanto no resto dos estados da união ele decresceu, variou de $-3,7 \%$. No mês de março, as variações foram de $10,8 \%$ e $5,6 \%$, respectivamente, em fevereiro foram de $-0,75 \%$ e $1,5 \%$ e em janeiro foram de $2,1 \%$ e $1,0 \% 2^{2}$. Considerando o Brasil todo, as variações mensais dos registros de óbitos foram de 1,6\% em janeiro, 0,35\% em fevereiro, 8,2\% em março, 9,76\% em abril e 20,63\% em maio de 2020 relativamente aos mesmos meses em 2019. Obviamente, há vários efeitos misturados nesses dados que merecem outras análises pormenorizadas. Porém, em estados com poucos casos de COVID-19 no mês de abril e que implementaram alguma medida de distanciamento social vemos uma redução significativa das mortes, enquanto nos estados amplamente noticiados como atingidos pela epidemia, vemos um aumento significativo.

Em maio, os números indicam um aumento de 20,63\% no número total de óbitos e um aumento de $77,93 \%$ no número de óbitos devido a problemas respiratórios em relação ao mesmo mês do ano passado. Esses valores podem ser provisórios e devem aumentar devido ao atraso entre os óbitos e seus registros. Assim, estatísticas segregadas por estados devem ser calculadas decorridos um tempo a mais. Porém, vemos claramente que dentre os 27 estados da federação, em somente 1/3 deles (GO, MG, MS, MT, PI, PR, RS, SC, TO) vemos uma redução na mortalidade (resultado esperado do distanciamento social).

Já as Figuras 2 até 10 apresentam o número de mortes pelas mesmas doenças respiratórias, segregados por estados. O impacto da COVID-19 nos números de óbitos nesses estados é facilmente observado.

\footnotetext{
${ }^{2}$ Esses números foram obtidos com os dados do portal em 05 de junho de 2020. Cumpre observar que como mencionado acima, eles podem variar levemente se computados entre diferentes dias devido à atualização de dados do portal não só do passado recente, mas de anos anteriores.
} 
Mortes (diárias) no Brasil entre janeiro e maio, $2019 \times 2020$
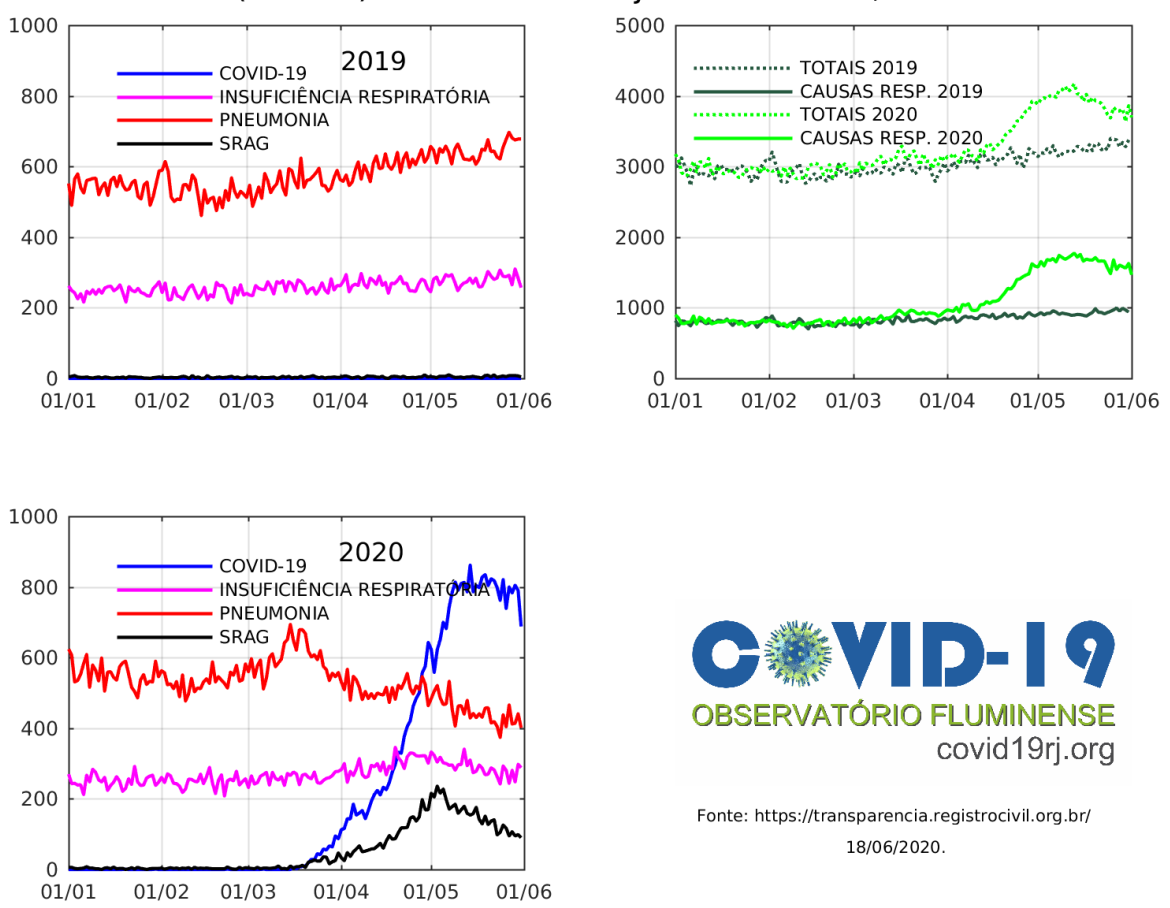

Fonte: https://transparencia.registrocivil.org.br/ $18 / 06 / 2020$.

Figura 1: Comparativo dos óbitos no Brasil entre os meses de janeiro a maio de 2020 relativamente a 2019.
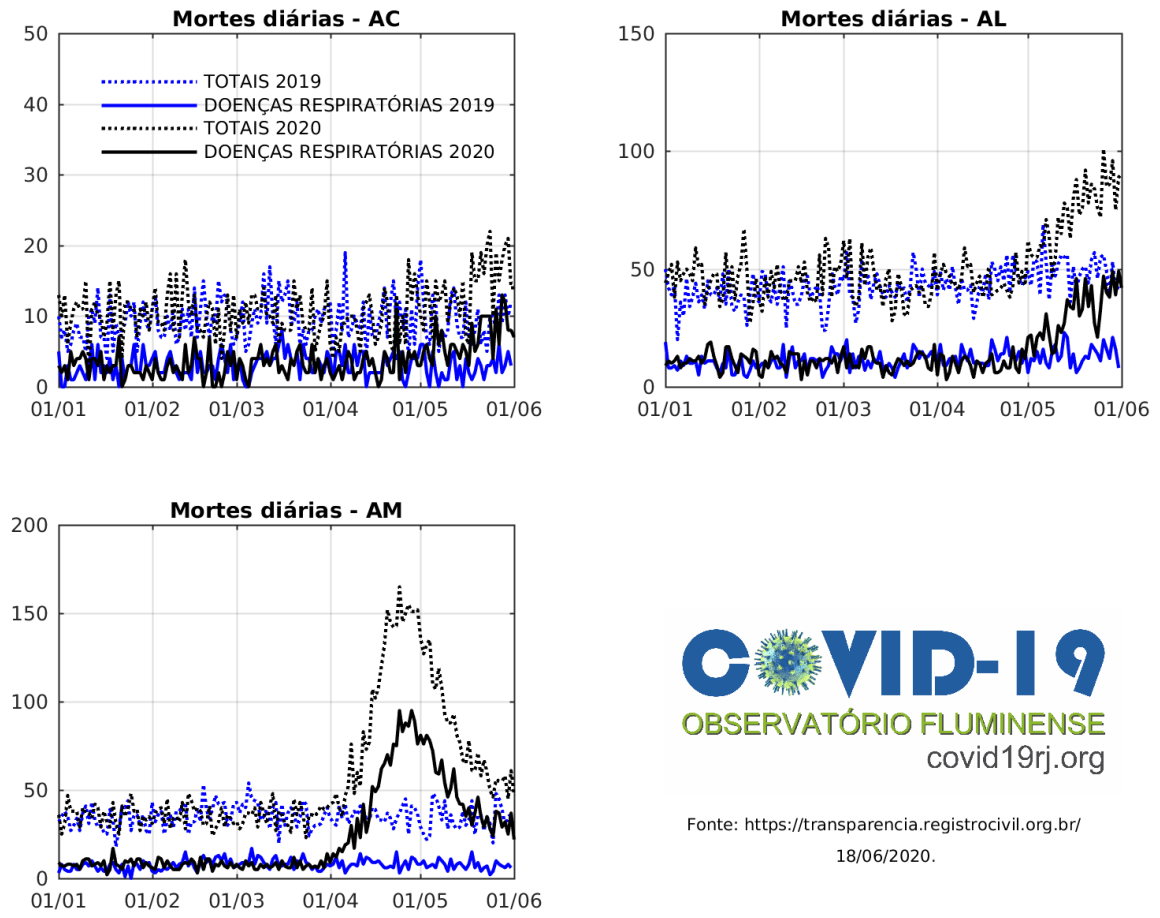

Fonte: https://transparencia.registrocivil.org.br/ $18 / 06 / 2020$.

Figura 2: Comparativo dos óbitos nos estados do Acre, Alagoas e Amazonas entre os meses de janeiro a maio de 2020 relativamente a 2019 . 

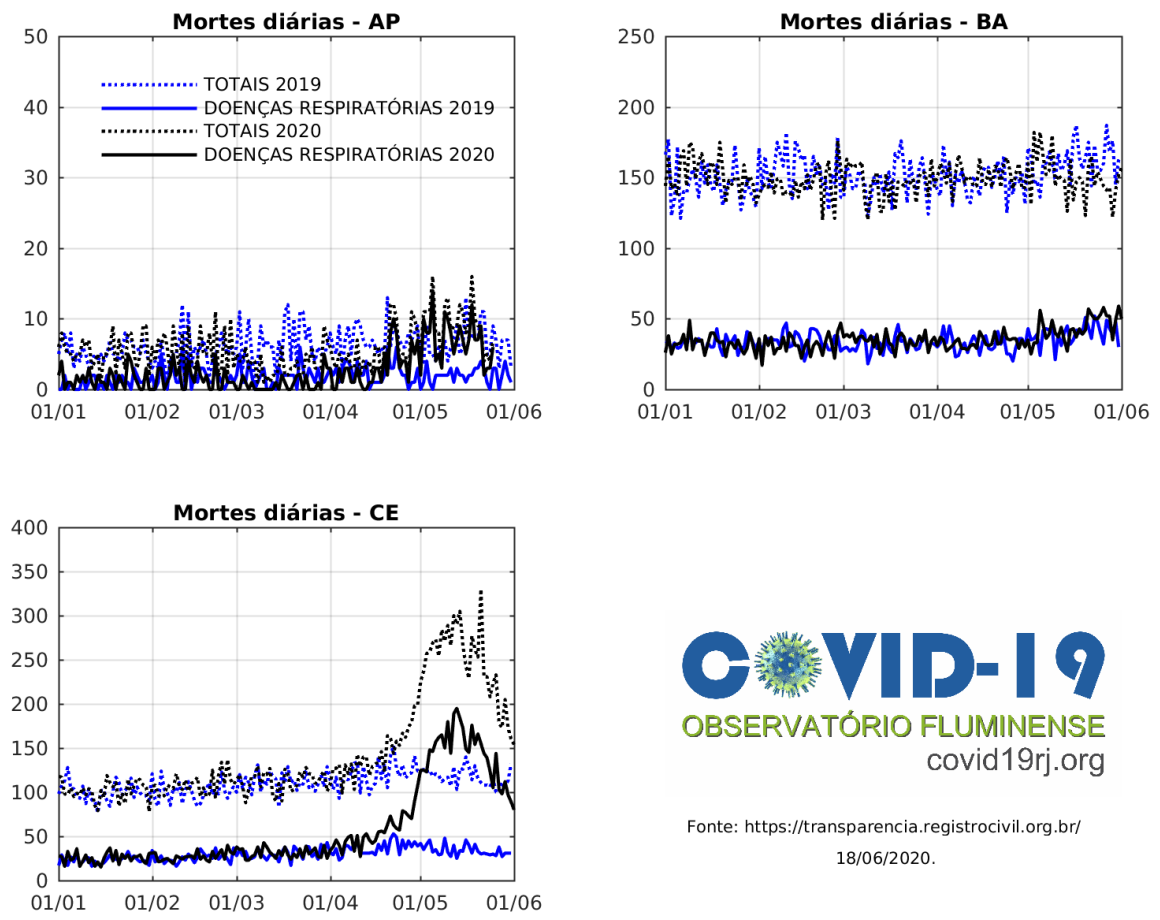

Fonte: https://transparencia.registrocivil.org.br/ $18 / 06 / 2020$.

Figura 3: Comparativo dos óbitos nos estados do Amapá, Bahia e Ceará entre os meses de janeiro a maio de 2020 relativamente a 2019 .
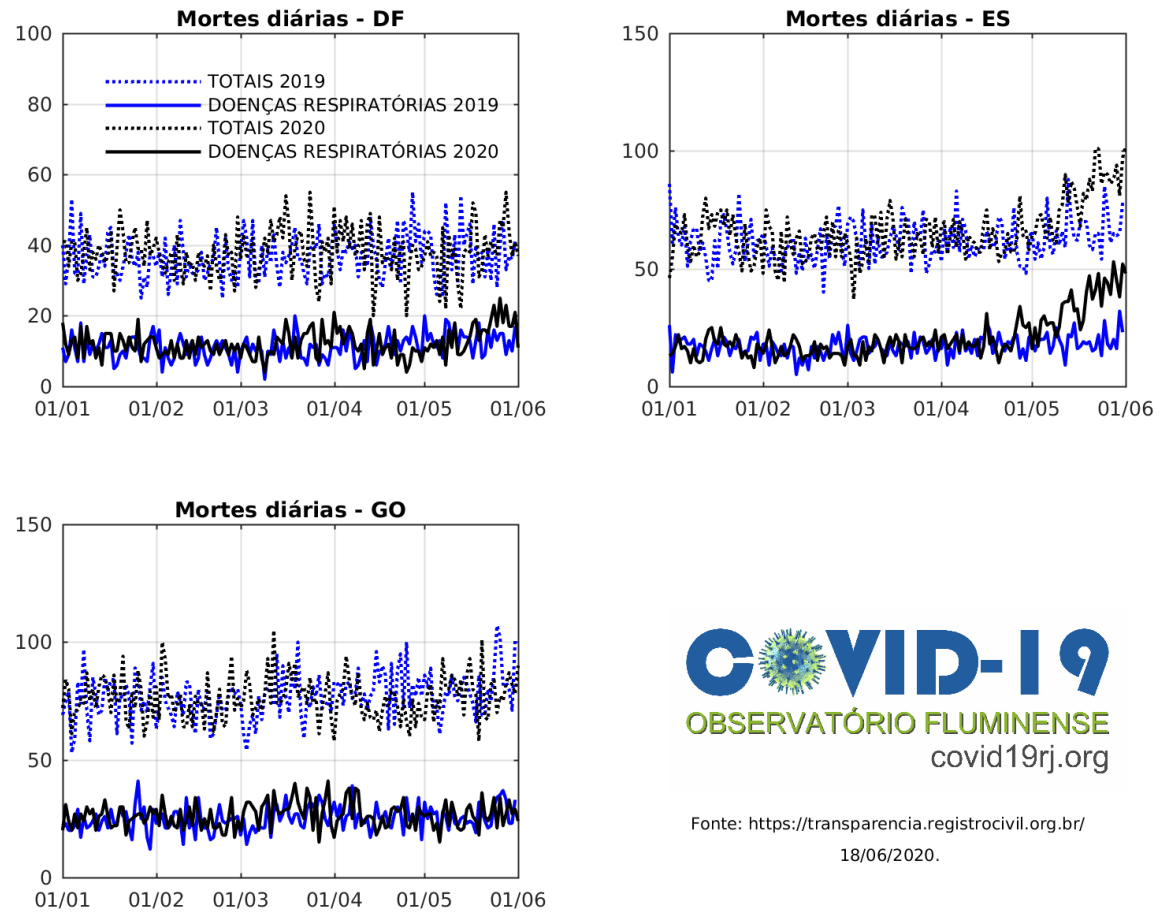

Fonte: https://transparencia.registrocivil.org.br/ $18 / 06 / 2020$.

Figura 4: Comparativo dos óbitos no Distrito Federal e nos estados do Espírito Santo e Goiás entre os meses de janeiro a maio de 2020 relativamente a 2019. 

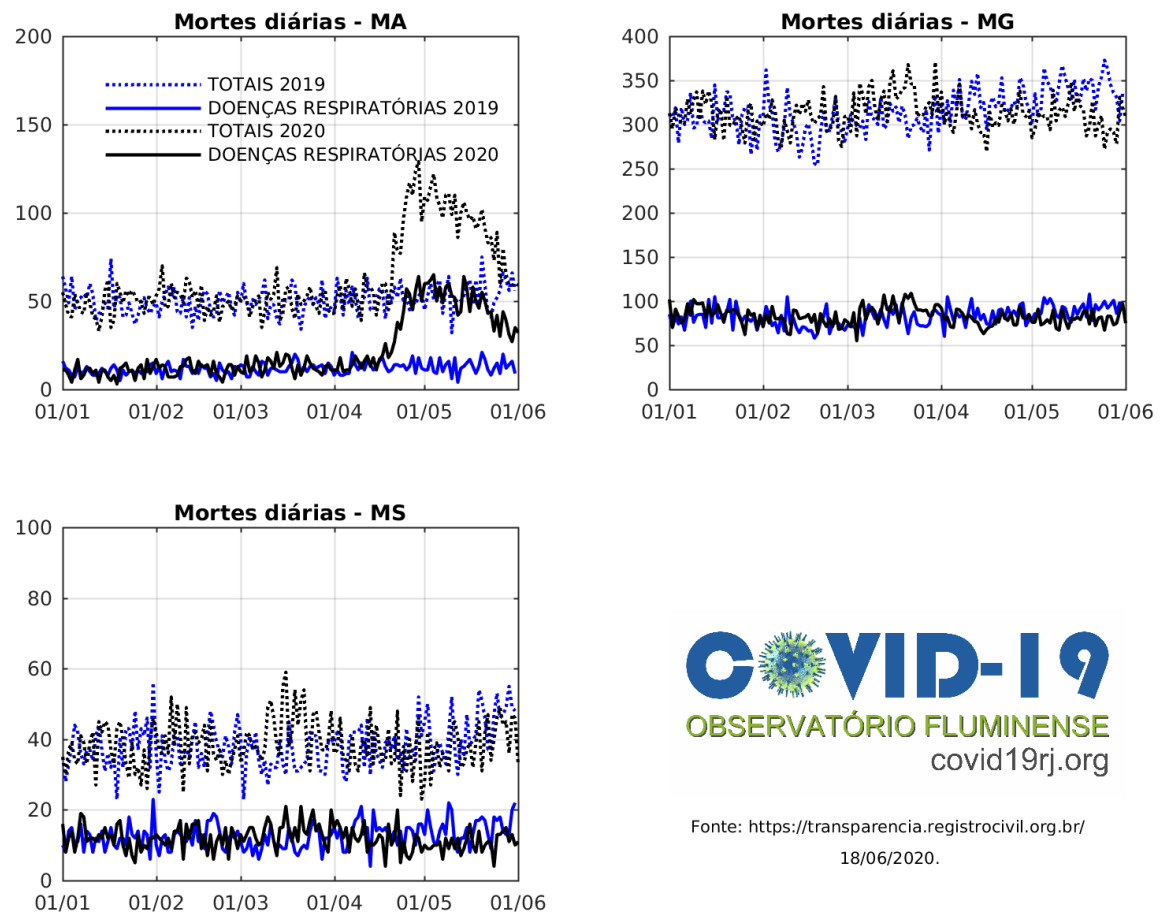

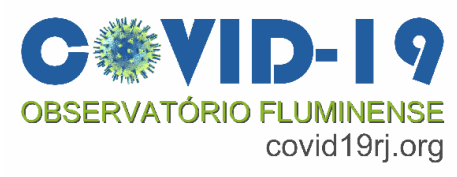

Fonte: https://transparencia.registrocivil.org.br/ 18/06/2020.

Figura 5: Comparativo dos óbitos nos estados do Maranhão, Minas Gerais e Mato-Grosso do Sul entre os meses de janeiro a maio de 2020 relativamente a 2019 .
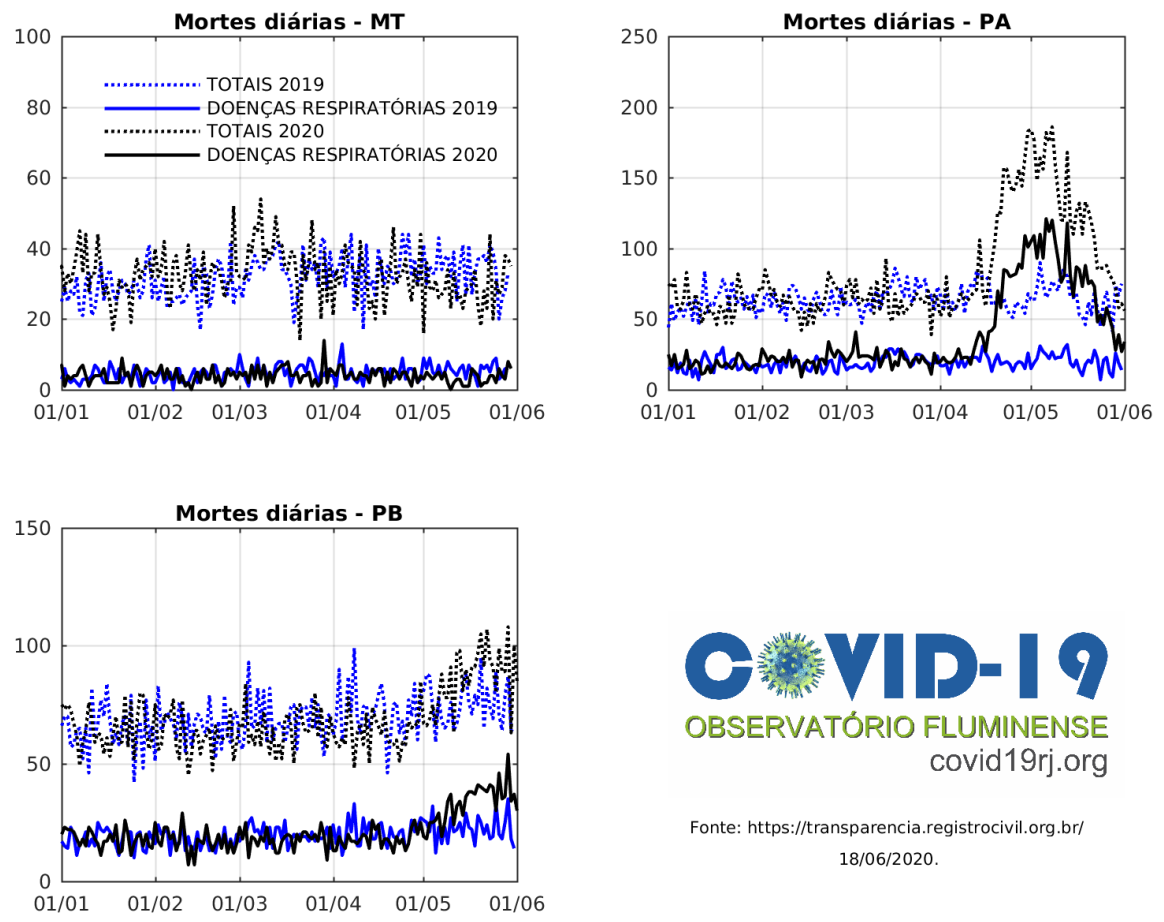

Fonte: https://transparencia.registrocivil.org.br/ 18/06/2020.

Figura 6: Comparativo dos óbitos nos estados do Mato-Grosso, Pará e Paraíba entre os meses de janeiro a maio de 2020 relativamente a 2019. 

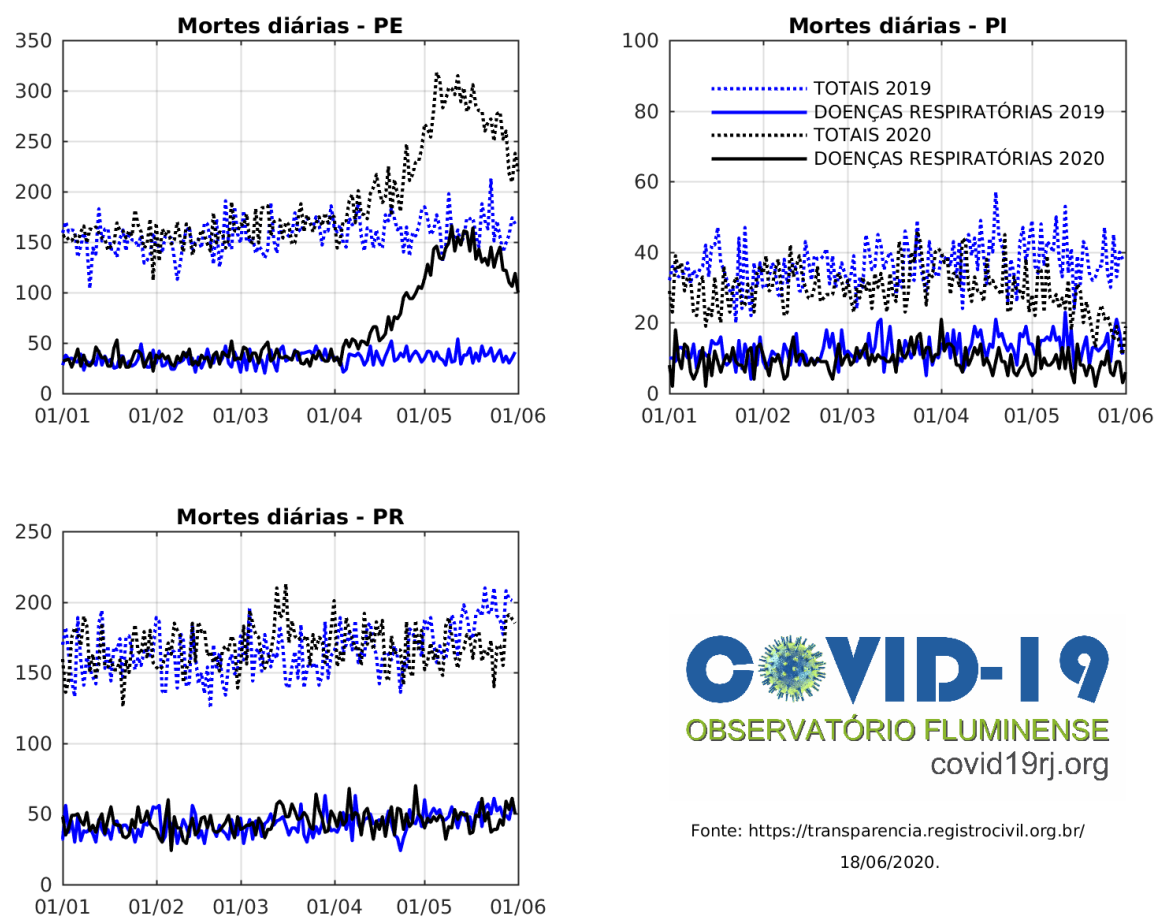

Fonte: https://transparencia.registrocivil.org.br/ 18/06/2020.

Figura 7: Comparativo dos óbitos nos estados de Pernambuco, Piauí e Paraná entre os meses de janeiro a maio de 2020 relativamente a 2019.
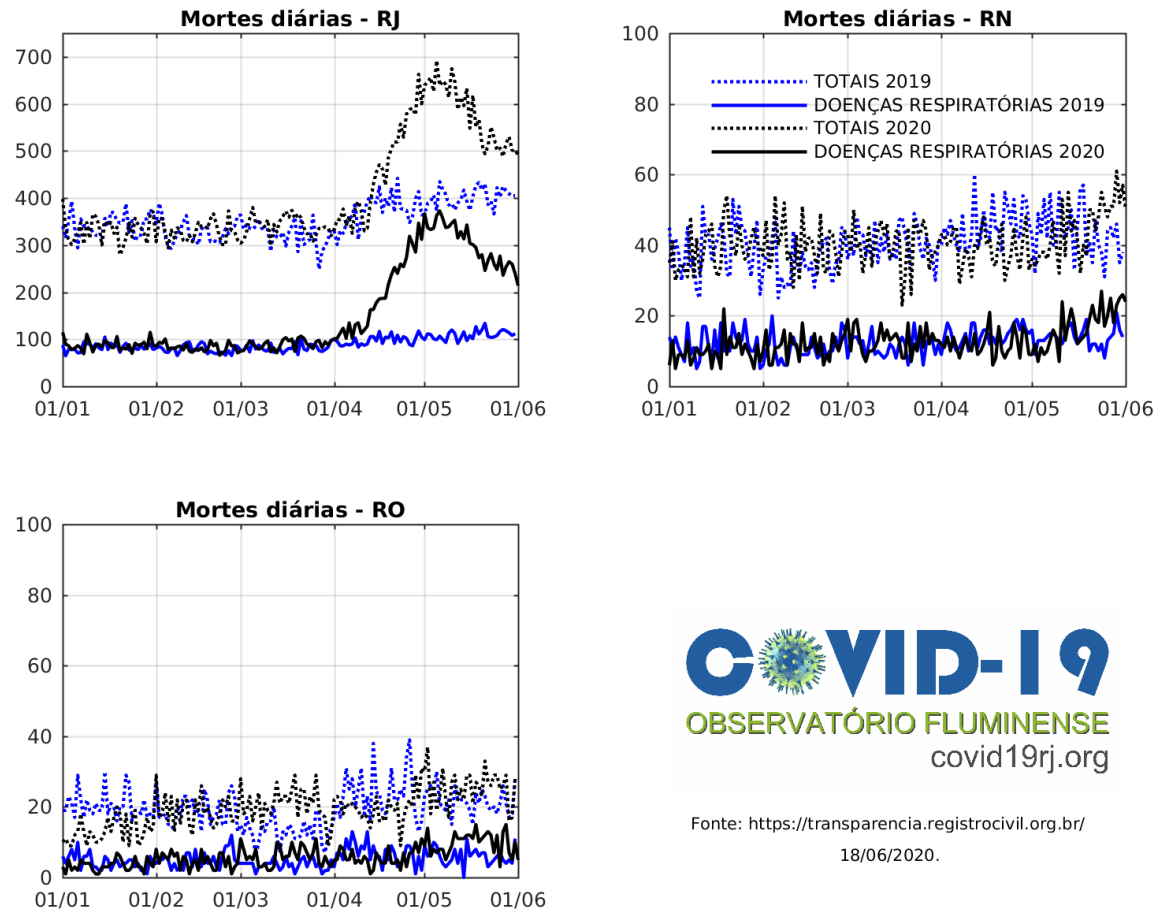

Fonte: https://transparencia.registrocivil.org.br/ 18/06/2020.

Figura 8: Comparativo dos óbitos nos estados do Rio de Janeiro, Rio Grande do Norte e Rondônia entre os meses de janeiro a maio de 2020 relativamente a 2019 . 

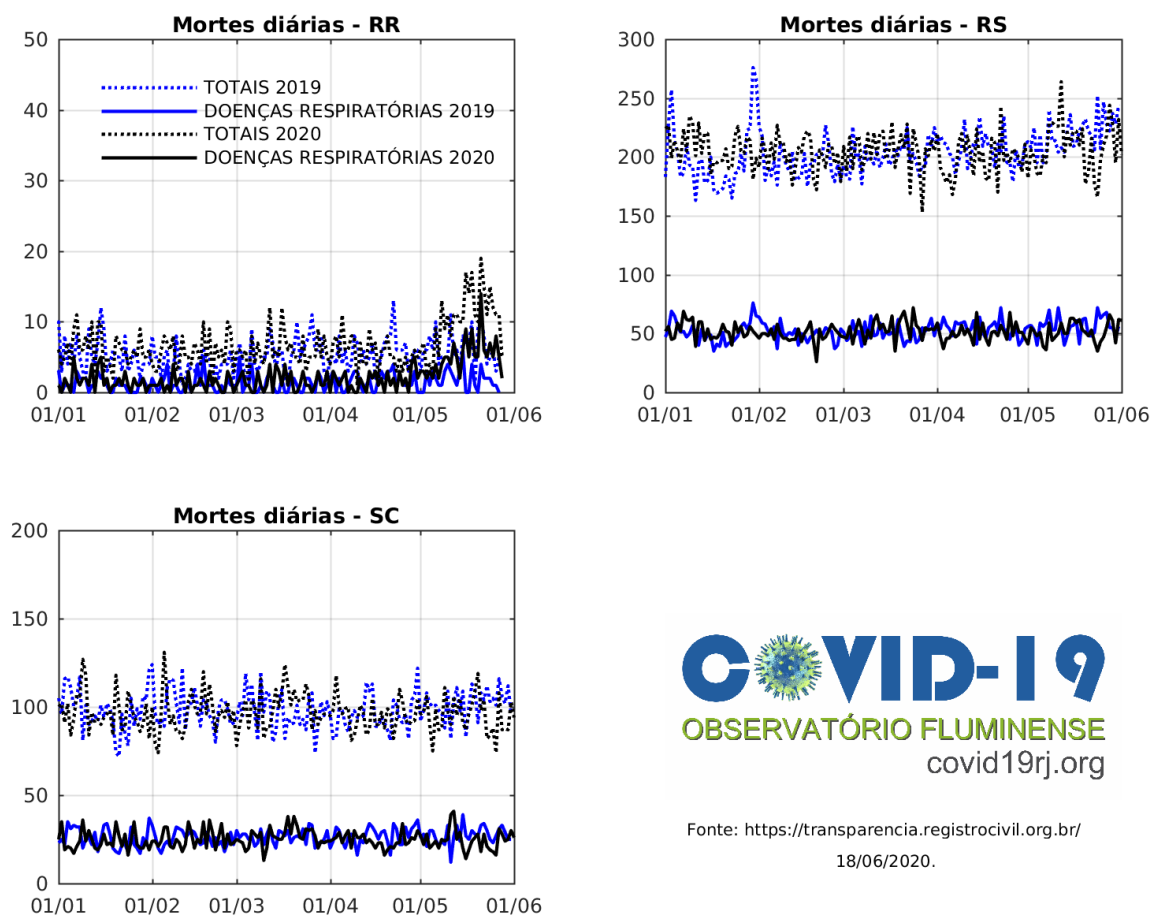

Fonte: https://transparencia.registrocivil.org.br/ 18/06/2020.

Figura 9: Comparativo dos óbitos nos estados de Roraima, Rio Grande do Sul e Santa Catarina entre os meses de janeiro a maio de 2020 relativamente a 2019.
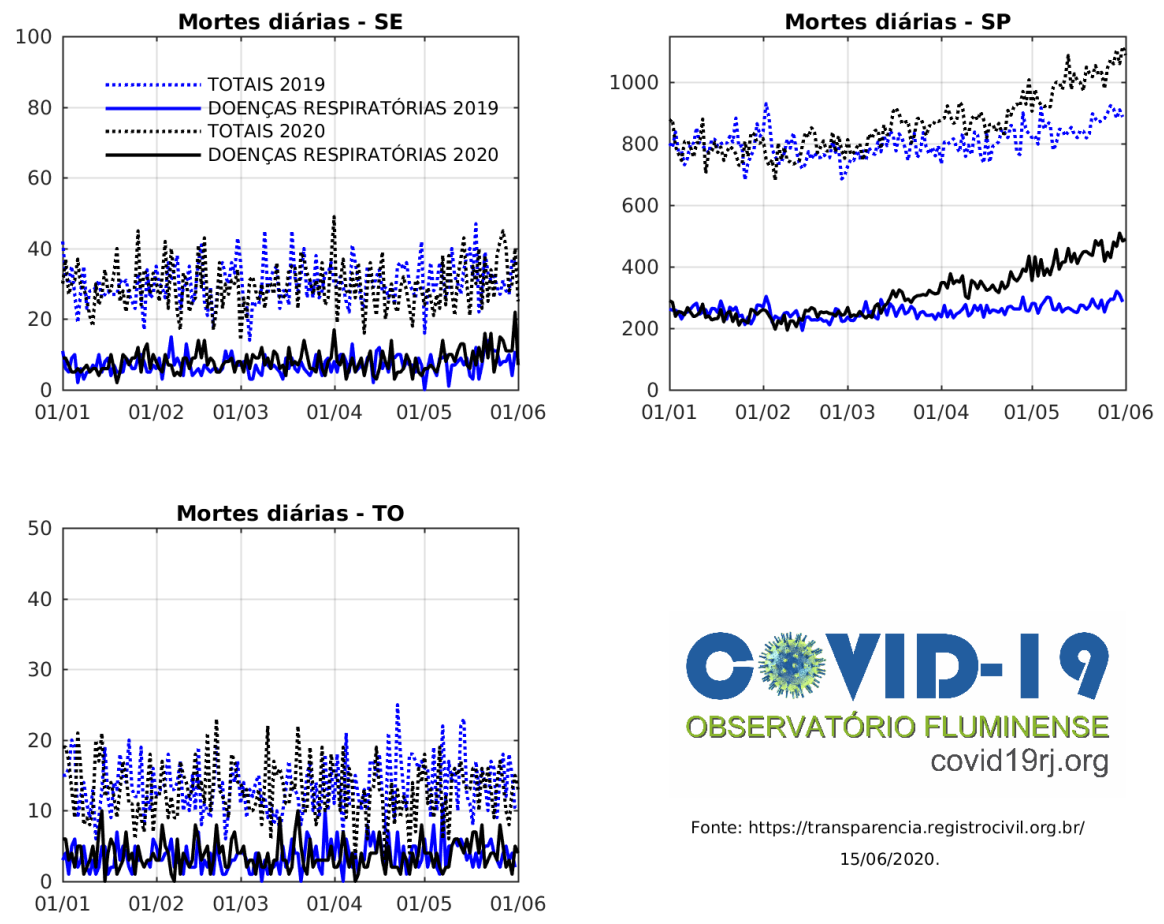

Fonte: https://transparencia.registrocivil.org.br/ 15/06/2020.

Figura 10: Comparativo dos óbitos nos estados de Sergipe, São Paulo e Tocantins entre os meses de janeiro a maio de 2020 relativamente a 2019 . 
Tabela 1: Números de mortes totais e por causas respiratórios nos meses de abril e maio de 2019 e 2020 e suas variações percentuais entre 2019 e 2020 - dados de 18 de junho de 2020 (continua na próxima página).

\begin{tabular}{|c|c|c|c|c|c|}
\hline Estado & Tipo & Mês & 2019 & 2020 & Variação (\% entre 20919 e 2020) \\
\hline \multirow{4}{*}{ BR } & \multirow{2}{*}{ Totais } & Abril & 92951 & 102021 & 9.758 \\
\hline & & Maio & 100966 & 121800 & 20.63 \\
\hline & \multirow{2}{*}{ Causa Resp. } & Abril & 26158 & 35624 & 36.19 \\
\hline & & Maio & 28826 & 51288 & 77.92 \\
\hline \multirow{4}{*}{$A C$} & \multirow{2}{*}{ Totais } & Abril & 290 & 286 & -1.379 \\
\hline & & Maio & 293 & 440 & 50.17 \\
\hline & \multirow{2}{*}{ Causa Resp. } & Abril & 98 & 95 & -3.061 \\
\hline & & Maio & 86 & 214 & 148.8 \\
\hline \multirow{4}{*}{$A L$} & \multirow{2}{*}{ Totais } & Abril & 1327 & 1375 & 3.617 \\
\hline & & Maio & 1529 & 2302 & 50.56 \\
\hline & \multirow{2}{*}{ Causa Resp. } & Abril & 379 & 302 & -20.32 \\
\hline & & Maio & 413 & 978 & 136.8 \\
\hline \multirow{4}{*}{ AM } & \multirow{2}{*}{ Totais } & Abril & 1070 & 3090 & 188.8 \\
\hline & & Maio & 1051 & 2419 & 130.2 \\
\hline & \multirow{2}{*}{ Causa Resp. } & Abril & 252 & 1561 & 519.4 \\
\hline & & Maio & 230 & 1395 & 506.5 \\
\hline \multirow{4}{*}{ AP } & \multirow{2}{*}{ Totais } & Abril & 215 & 151 & -29.77 \\
\hline & & Maio & 202 & 269 & 33.17 \\
\hline & \multirow{2}{*}{ Causa Resp. } & Abril & 72 & 73 & 1.389 \\
\hline & & Maio & 61 & 200 & 227.9 \\
\hline \multirow{4}{*}{ BA } & \multirow{2}{*}{ Totais } & Abril & 4425 & 4555 & 2.938 \\
\hline & & Maio & 5009 & 4660 & -6.967 \\
\hline & \multirow{2}{*}{ Causa Resp. } & Abril & 958 & 1002 & 4.593 \\
\hline & & Maio & 1225 & 1364 & 11.35 \\
\hline \multirow{4}{*}{ CE } & \multirow{2}{*}{ Totais } & Abril & 3598 & 4391 & 22.04 \\
\hline & & Maio & 3633 & 7584 & 108.8 \\
\hline & \multirow{2}{*}{ Causa Resp. } & Abril & 1107 & 1744 & 57.54 \\
\hline & & Maio & 1056 & 4421 & 318.7 \\
\hline \multirow{4}{*}{ DF } & Totais & Abril & 1168 & 1140 & -2.397 \\
\hline & Iotals & Maio & 1176 & 1197 & 1.786 \\
\hline & Санса Resn & Abril & 345 & 332 & -3.768 \\
\hline & Causa Resp. & Maio & 418 & 462 & 10.53 \\
\hline & Totais & Abril & 1835 & 1885 & 2.725 \\
\hline ES & Totals & Maio & 2028 & 2519 & 24.21 \\
\hline ES & Слисл Rесп & Abril & 533 & 589 & 10.51 \\
\hline & Causa Resp. & Maio & 597 & 1103 & 84.76 \\
\hline & Totais & Abril & 2429 & 2194 & -9.675 \\
\hline$G \Omega$ & & Maio & 2481 & 2467 & -0.5643 \\
\hline U & Саиса Resn & Abril & 773 & 805 & 4.14 \\
\hline & Causa Resp. & Maio & 821 & 807 & -1.705 \\
\hline & Totais & Abril & 1546 & 2155 & 39.39 \\
\hline$M A$ & Iotais & Maio & 1688 & 2820 & 67.06 \\
\hline IVIA & Causa Resp & Abril & 384 & 829 & 115.9 \\
\hline & Causa Resp. & Maio & 401 & 1477 & 268.3 \\
\hline & Totais & Abril & 9768 & 9209 & -5.723 \\
\hline$M G$ & & Maio & 10506 & 9570 & -8.909 \\
\hline IVIG & Санса Resn & Abril & 2533 & 2362 & -6.751 \\
\hline & Causa Resp. & Maio & 2828 & 2473 & -12.55 \\
\hline
\end{tabular}


Tabela 1: Números de mortes totais e por causas respiratórios nos meses de abril e maio de 2019 e 2020 e suas variações percentuais entre 2019 e 2020 - dados de 18 de junho de 2020 (continuação da página anterior).

\begin{tabular}{|c|c|c|c|c|c|}
\hline Estado & Tipo & Mês & 2019 & 2020 & Variação (\% entre 20919 e 2020) \\
\hline \multirow{4}{*}{ MS } & \multirow{2}{*}{ Totais } & Abril & 1143 & 1088 & -4.812 \\
\hline & & Maio & 1330 & 1206 & -9.323 \\
\hline & \multirow{2}{*}{ Causa Resp. } & Abril & 407 & 342 & -15.97 \\
\hline & & Maio & 458 & 329 & -28.17 \\
\hline \multirow{4}{*}{ MT } & \multirow{2}{*}{ Totais } & Abril & 1018 & 952 & -6.483 \\
\hline & & Maio & 1056 & 906 & -14.2 \\
\hline & \multirow{2}{*}{ Causa Resp. } & Abril & 147 & 128 & -12.93 \\
\hline & & Maio & 171 & 106 & -38.01 \\
\hline \multirow{4}{*}{ PA } & \multirow{2}{*}{ Totais } & Abril & 1954 & 3099 & 58.6 \\
\hline & & Maio & 2050 & 3729 & 81.9 \\
\hline & \multirow{2}{*}{ Causa Resp. } & Abril & 590 & 1545 & 161.9 \\
\hline & & Maio & 636 & 2362 & 271.4 \\
\hline \multirow{4}{*}{ PB } & \multirow{2}{*}{ Totais } & Abril & 2148 & 1904 & -11.36 \\
\hline & & Maio & 2323 & 2681 & 15.41 \\
\hline & \multirow{2}{*}{ Causa Resp. } & Abril & 621 & 548 & -11.76 \\
\hline & & Maio & 694 & 1046 & 50.72 \\
\hline \multirow{4}{*}{ PE } & \multirow{2}{*}{ Totais } & Abril & 4844 & 5972 & 23.29 \\
\hline & & Maio & 5095 & 8528 & 67.38 \\
\hline & \multirow{2}{*}{ Causa Resp. } & Abril & 1081 & 2042 & 88.9 \\
\hline & & Maio & 1155 & 4227 & 266 \\
\hline \multirow{4}{*}{ PI } & \multirow{2}{*}{ Totais } & Abril & 1178 & 948 & -19.52 \\
\hline & & Maio & 1193 & 729 & -38.89 \\
\hline & \multirow{2}{*}{ Causa Resp. } & Abril & 422 & 280 & -33.65 \\
\hline & & Maio & 435 & 249 & -42.76 \\
\hline \multirow{4}{*}{ PR } & \multirow{2}{*}{ Totais } & Abril & 5003 & 5086 & 1.659 \\
\hline & & Maio & 5717 & 5214 & -8.798 \\
\hline & \multirow{2}{*}{ Causa Resp. } & Abril & 1282 & 1408 & 9.828 \\
\hline & & Maio & 1544 & 1435 & -7.06 \\
\hline \multirow{4}{*}{ RJ } & & Abril & 11186 & 13913 & 24.38 \\
\hline & Totais & Maio & 12445 & 17834 & 43.3 \\
\hline & Crusa Rosn & Abril & 2931 & 6036 & 105.9 \\
\hline & Causa resp. & Maio & 3388 & 9277 & 173.8 \\
\hline & Totaic & Abril & 1366 & 1169 & -14.42 \\
\hline & lotals & Maio & 1343 & 1396 & 3.946 \\
\hline RN & & Abril & 434 & 356 & -17.97 \\
\hline & Causa Resp. & Maio & 429 & 532 & 24.01 \\
\hline & & Abril & 707 & 602 & -14.85 \\
\hline $\mathrm{RO}$ & Totais & Maio & 674 & 787 & 16.77 \\
\hline RO & Сәисз Reсn & Abril & 203 & 172 & -15.27 \\
\hline & Causa Resp. & Maio & 189 & 303 & 60.32 \\
\hline & Totric & Abril & 171 & 170 & -0.5848 \\
\hline RR & lotais & Maio & 186 & 304 & 63.44 \\
\hline $\mathrm{RR}$ & Coura Porn & Abril & 48 & 43 & -10.42 \\
\hline & Causa Resp. & Maio & 55 & 153 & 178.2 \\
\hline & & Abril & 6175 & 5942 & -3.773 \\
\hline$R c^{2}>$ & Totais & Maio & 6811 & 6506 & -4.478 \\
\hline RS & Саиса Resn & Abril & 1637 & 1500 & -8.369 \\
\hline & Causa Resp. & Maio & 1742 & 1607 & -7.75 \\
\hline
\end{tabular}


Tabela 1: Números de mortes totais e por causas respiratórios nos meses de abril e maio de 2019 e 2020 e suas variações percentuais entre 2019 e 2020 - dados de 18 de junho de 2020 (continuação da página anterior).

\begin{tabular}{|c|c|c|c|c|c|}
\hline Estado & Tipo & Mês & 2019 & 2020 & Variação (\% entre 20919 e 2020) \\
\hline \multirow{4}{*}{ SC } & \multirow{2}{*}{ Totais } & Abril & 2875 & 2859 & -0.5565 \\
\hline & & Maio & 3157 & 2947 & -6.652 \\
\hline & \multirow{2}{*}{ Causa Resp. } & Abril & 808 & 736 & -8.911 \\
\hline & & Maio & 847 & 759 & -10.39 \\
\hline \multirow{4}{*}{ SE } & \multirow{2}{*}{ Totais } & Abril & 924 & 827 & -10.5 \\
\hline & & Maio & 981 & 977 & -0.4077 \\
\hline & \multirow{2}{*}{ Causa Resp. } & Abril & 232 & 203 & -12.5 \\
\hline & & Maio & 240 & 316 & 31.67 \\
\hline \multirow{4}{*}{ SP } & \multirow{2}{*}{ Totais } & Abril & 24155 & 26710 & 10.58 \\
\hline & & Maio & 26560 & 31407 & 18.25 \\
\hline & \multirow{2}{*}{ Causa Resp. } & Abril & 7764 & 10495 & 35.18 \\
\hline & & Maio & 8577 & 13567 & 58.18 \\
\hline \multirow{4}{*}{ TO } & \multirow{2}{*}{ Totais } & Abril & 433 & 349 & -19.4 \\
\hline & & Maio & 449 & 404 & -10.02 \\
\hline & \multirow{2}{*}{ Causa Resp. } & Abril & 117 & 96 & -17.95 \\
\hline & & Maio & 130 & 127 & -2.308 \\
\hline
\end{tabular}

\section{Referências}

[1] Portal da Transparência de Registro Civil, 2020. https://transparencia.registrocivil.org.br.

[2] A. Cunha Jr and et al. Relatório 01 Progresso da COVID-19 no Brasil e no Estado do Rio de Janeiro $21^{a}$ Semana Epidemiológica do Calendário 2020 (17/5/2020 até 23/5/2020). COVID-19: Observatório Fluminense, (24-05-2020). https://https://doi.org/10.12957/eduerj.covid19rj.relatorio1. 